\title{
Vergleichbare Wirksamkeit von Pazopanib und Sunitinib
}

\begin{abstract}
Die Ergebnisse der Studie COMPARZ (Comparing the Efficacy, Safety and Tolerability of Pazopanib vs. Sunitinib in first-line advanced and/or metastatic Renal Cell Carcinoma), einer direkten Vergleichsstudie mit den beiden Tyrosinkinasehemmern Pazopanib und Sunitinib als Erstlinien-Behandlung bei Patienten mit fortgeschrittenem bzw. metastasiertem Nierenzellkarzinom, belegen eine vergleichbare Wirksamkeit der beiden Substanzen. Die PhaseIII-Studie wurde als Nicht-Unterlegenheitsstudie angelegt.
\end{abstract}

In der Studie wurden 1.100 Patienten 1:1 randomisiert (Motzer R et al., ESMO 2012: LBA8). Primärer Endpunkt, um die NichtUnterlegenheit von Pazopanib im Vergleich zu Sunitinib zu zeigen, war die progressionsfreie Überlebenszeit (PFS). Alle randomisierten Patienten hatten ein fortgeschrittenes bzw. metastasiertes Nierenzellkar- im Vorfeld festgelegte 95\%-Konfidenzintervall für den Nachweis der Nicht-Unterlegenheit wurde nicht überschritten. Im Median blieben die Patienten 8,4 Monate (Pazopanib) bzw. 9,5 Monate (Sunitinib) ohne erneuten Progress (Hazard-Ratio 1,047). „Aus klinischer Sicht“, so Motzer, „bin ich sehr überzeugt, dass wir von einer vergleichbaren Wirksamkeit beider Substanzen ausgehen können." Die Ansprechraten bestätigen dies. Eine objektive Remission erreichten $31 \%$ (Pazopanib) bzw. 25\% (Sunitinib) der Patienten ( $\mathrm{p}=0,032)$. Zuzüglich der Krankheitsstabilisierungen sprachen $70 \%$ bzw. 69\% der Patienten auf die Behandlung mit Pazopanib bzw. Sunitinib an. Die mediane Gesamtüberlebenszeit basiert derzeit auf 554 von 631 für die Endanalyse geforderten Ereignissen. Sie beträgt in beiden Patientengruppen bereits deutlich mehr als zwei Jahre: 28,4 bzw. 29,3 Monate im Pazopanib- bzw. Sunitinib-Arm (Hazard-Ratio 0,908; $\mathrm{p}=0,275)$.

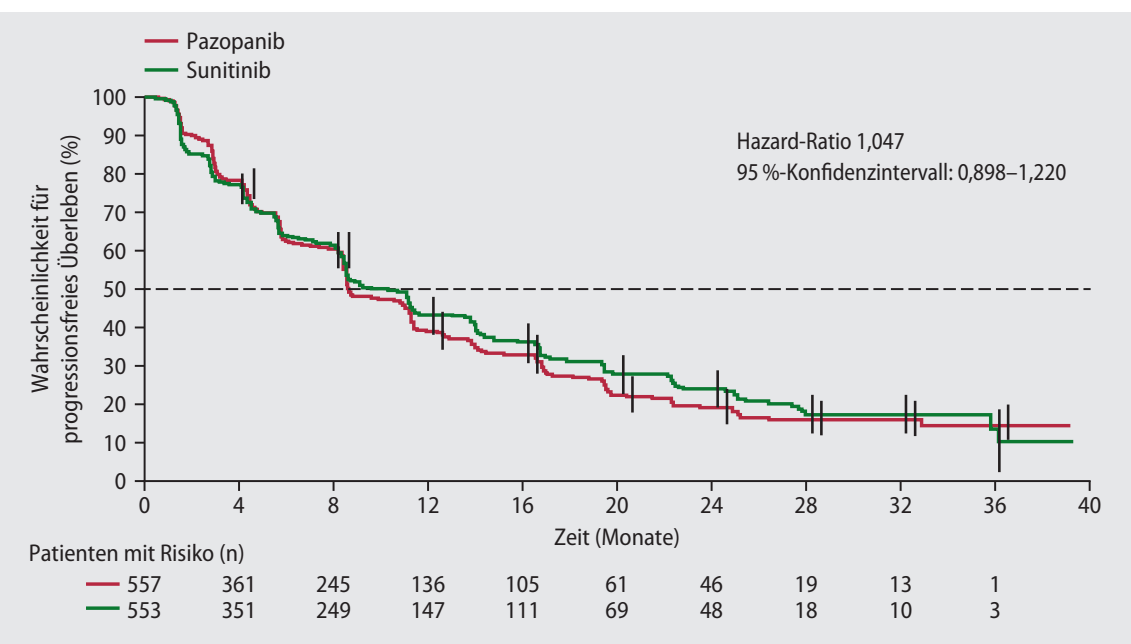

Progressionsfreies Überleben: vergleichbare Wirksamkeit von Pazopanib und Sunitinib (nach Motzer R et al., ESMO 2012:LBA8)

zinom mit klarzelliger Histologie, waren systemisch nicht vorbehandelt und hatten einen guten Allgemeinzustand, erläuterte Prof. Dr. Robert Motzer, New York/USA.

\section{Nicht-Unterlegenheit bestätigt}

Ergebnisse der Auswertung des PFS, die durch ein unabhängiges Expertengremium erfolgte, bestätigten eine vergleichbare Wirksamkeit beider Substanzen (Abb.). Das
Vorteile von Pazopanib (Votrient ${ }^{\circledR}$ ) zeigten sich beim Nebenwirkungsprofil, was auch mit einer besseren Lebensqualität der Patienten korrelierte, so Motzer. Vor allem Nebenwirkungen, wie z.B. Fatigue, Hautausschlag, Mukositis, Geschmackveränderungen oder das Hand-Fuß-Syndrom, waren unter Pazopanib seltener.

(pm)

Satellitensymposium der GlaxoSmithKline GmbH \& Co. KG

\section{Dreijährige adjuvante Imatinib-Therapie bei GIST}

Die European Society for Medical Oncology (ESMO) hat im September 2012 ihre Leitlinien zur Diagnose, Therapie und Nachsorge von Patienten mit gastrointestinalen Stromatumoren (GIST) aktualisiert (The ESMO/ European Sarcoma Network Working Group, Ann Oncol 2012, 23 [Suppl 7]:4955). Diese empfehlen bei Patienten mit primär operablen GIST und hohem Rezidivrisiko als Standard eine dreijährige adjuvante Therapie mit Imatinib. Basis dafür sind die Daten der StudieSSGXVIII/AIO zum Vergleich einer 36- bzw. 12-monatigen adjuvanten Therapie mit Imatinib (Glivec ${ }^{\circledR}$ ) bei Hochrisikopatienten nach kompletter Resektion c-KIT-positiver GIST (Joensuu $\mathrm{H}$ et al., JAMA 2012, 307:1265-1272): Fünf Jahre nach Therapiebeginn waren 65,6\% der Patienten unter dreijähriger Therapie im Vergleich zu 47,9\% unter einjähriger Behandlung rezidivfrei $(p<0,0001)$. Zudem verlängerte sich unter 36-monatiger Therapie das Gesamtüberleben signifikant $(p=0,019)$.

nach Informationen der Novartis Pharma GmbH

\section{Verlängertes PFS bei neu diagnostiziertem Glioblastom}

Wie Daten der Phase-III-Studie AVAglio belegen, kann das mediane progressionsfreie Überleben bei Patienten mit neu diagnostiziertem Glioblastom unter der Behandlung mit Bevacizumab (Avastin $^{\circledast}$ ) in Kombination mit Bestrahlung und Chemotherapie im Vergleich zur Bestrahlung und Chemotherapie allein um 4,4 Monate verlängert werden $(p<0,0001)$ (Chinot $O$ et al., SNO 2012:\#OT03). Das Risiko des Fortschreitens der Erkrankung wurde in der Studie um $36 \%$ reduziert (Hazard-Ratio 0,64; $\mathrm{p}<0,0001$ ).

Neue Sicherheitsbefunde wurden nicht beobachtet und die Nebenwirkungen entsprachen denen in früheren Studien mit Bevacizumab bei verschiedenen Tumorarten in den zugelassenen Indikationen. 